\title{
Aproximaciones interculturales
}

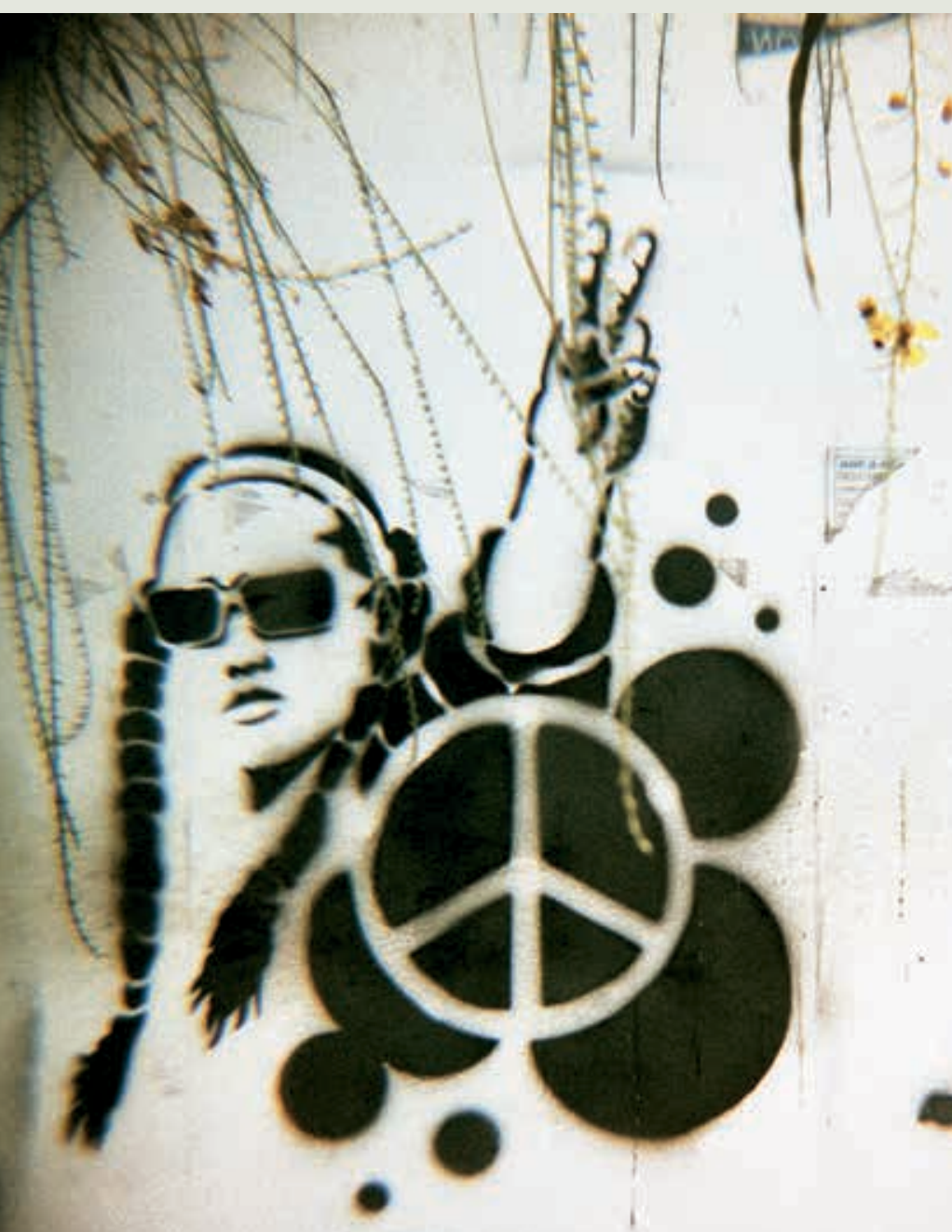




\title{
Hacia la consolidación de los estudios de consumo cultural en Latinoamérica
}

\author{
Carlos Enrique Guzmán Cárdenas \\ (Ininco, Universidad Central de Venezuela, Venezuela)
}

Recibido: $26 / 8 / 2015$

Aprobado: 25/9/2015

\begin{abstract}
Resumen. El consumo cultural como problema objeto de estudio desde la mirada de los públicos, audiencias o receptores es reciente en América Latina y el Caribe. En los últimos veinticinco años, indudablemente se ha generado un conjunto de proposiciones teóricas con sustento empírico, influenciados por los estudios culturales, la semiótica, la antropología, la sociología de la cultura y la economía política respecto de los modos en que los públicos ven, escuchan y leen; los usos que les dan a los bienes y servicios culturales; las maneras en que ellos relacionan esos bienes con su vida cotidiana, hasta el conjunto de teorías más importantes sobre la estratificación social en el consumo cultural.
\end{abstract}

Palabras clave: creación / cultura / consumo cultural / demanda cultural / estudios / encuestas / Iberoamérica

\section{Towards the Consolidation of the Studies of Cultural Consumption in Latin America}

Abstract. As object of study, cultural consumption from a reception perspective is a recent development in Latin America and the Caribbean. In the last twenty years, theoretical propositions stemming from empirical evidence have emerged influenced by cultural studies, semiotics, anthropology, sociology of culture, and political economy. These theories grapple with the ways in which public watch, listen and read; the uses the public gives to cultural goods and services; the ways in which the public relate these goods to their daily lives; as well with the most important theories of social stratification relative to cultural consumption.

Key words: creation / consumption / cultural consumption / cultural demand / studies / surveys / Latin America 


\section{Introducción}

Las personas mayores, las que viven en $\mathrm{ni}$ veles económicos y sociales bajos y las que tienen menos estudios participan de forma más limitada en las actividades culturales, tienen una menor valoración de la oferta existente y sus expectativas futuras son más reducidas. No es extraño, por tanto, que los países con menor desarrollo económico y educativo muestren, salvo excepciones que merece la pena analizar, una menor participación en las actividades culturales. (...) la cultura puede ser un lugar de encuentro y de inclusión social de todos los ciudadanos [negritas añadidas] y todos ellos la valoran de forma positiva. Sin embargo, aún se manifiestan notables diferencias por razones del nivel social y educativo de las personas (OEI, 2014, p. 14).

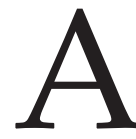

unque en la tradición norteamericana y en algunos países de Europa (particularmente Reino Unido, Francia y Alemania) los estudios de públicos, consumo cultural y economía de la cultura tienen una larga tradición, en el espacio cultural iberoamericano solo a partir de finales de los años ochenta comienzan a desarrollarse -de manera más o menos sistemática- investigaciones en este campo de las ciencias sociales, si asumimos como referencias académicas dos publicaciones representativas. La primera de ellas, realizada por el programa de la Facultad Latinoamericana de Ciencias Sociales (Flacso) en Santiago de Chile, denominada Algunas tendencias en el consumo cultural de bienes culturales en América Latina (1992), bajo la coordinación de los chilenos ${ }^{1}$ Carlos Catalán y Guillermo Sunkel. El análisis de los resultados de dicho estudio incorporó los datos obtenidos por una encuesta aplicada en las ciudades de Santiago de Chile en mayo de 1987; en Buenos Aires entre los meses de julio y septiembre de 1988; y en Ciudad de México en septiembre y octubre de 1989. El segundo estudio fue elaborado por la Sociedad General de Autores y Editores de España (SGAE) intitulado Informe SGAE sobre hábitos de consumo cultural. 1997-1998², dirigido por

1 De los mismos autores (1980), Consumo cultural en Chile: la elite, lo masivo y lo popular. Documento de trabajo Flacso. N. ${ }^{\circ} 455$. Chile. Recomendamos leer como texto fundacional de los estudios de consumo cultural en Chile, Brunner, J. J., Barrios, A., y Catalán, C. (1989). Chile: Transformaciones culturales y modernidad. Santiago de Chile: Facultad Latinoamericana de Ciencias Sociales/Flacso.

2 El objetivo del estudio fue presentar información sobre los patrones de consumo y perfil de los consumidores de bienes culturales, que sirva de apoyo a la toma de decisiones de política cultural y de inversión privada en el sector. La técnica e instrumento de investigación fue la aplicación de cuestionarios estructurados con entrevistas personales en el domicilio de los entrevistados. Encuestas realizadas en cuatro olas anuales en los 15 días posteriores a la conclusión de cada trimestre. La muestra fue de 24000 entrevistas a la población española de más de 14 años residentes en la Península e Islas Baleares y 
la Fundación Autor. Ciertamente, algunos desplazamientos fundamentales facilitaron el inicio y tratamiento conceptual en torno a lo que entenderemos por consumo cultural, pero las dificultades crecientes en las fuentes de financiamiento de las actividades culturales $^{3}$ exigían demostraciones de la importancia que las obras y los servicios culturales ${ }^{4}$ tenían para sus públicos; la identificación de políticas públicas que ubicaran a la cultura como un motor imprescindible del quehacer nacional, y por tanto la necesidad de acceder a estadísticas fiables que permitieran medir sus debilidades y fortalezas, así como el impacto en eso que los planificadores llaman 'poblaciones objetivo'; las exigencias sociales de un desarrollo cultural armónico, pluralista y equitativo que garantizara una mayor democratización en el acceso de los ciudadanos a los bienes y servicios culturales, y el reconocimiento de la importancia cada vez mayor de las implicaciones econó-

Canarias y, estratificadas por comunidades autónomas y hábitat en cada una de las olas. Con continuidad, hasta la fecha se han realizado las siguientes: Ministerio de Cultura de España, División de Estadísticas Culturales (2011). Encuesta de hábitos y prácticas culturales en España 2010-2011. España. Secretaría General Técnica. Subdirección General de Publicaciones, Información y Documentación; Ministerio de Cultura de España, División de Estadísticas Culturales (2007). Encuesta de hábitos y prácticas culturales en España 2006-2007. España. Secretaría General Técnica. Subdirección General de Publicaciones, Información y Documentación; Ministerio de Cultura de España. Encuesta de hábitos y prácticas culturales en España 2002-2003. España. Ministerio de Cultura y la Fundación Autor (del Grupo SGAE).

3 "El universo de las actividades culturales es muy grande; dentro de lo que consideramos como sector cultural están distintos tipos de actividades culturales: desde expresiones del folclor, la cultura popular y la cultura mediática, hasta manifestaciones de la cultura de 'élite' o 'bellas artes' y el patrimonio histórico. Las manifestaciones económicas que se hallan dentro de esta tipología también son variadas. Algunas se desarrollan en los mercados, otras son subvencionadas por el Estado o por mecenas; en muchos casos, las motivaciones de la creación residen en ámbitos distintos al de obtener ganancias y no necesariamente participan en dinámicas económicas de oferta y demanda, donde el valor económico se vea reflejado en un precio. Ingreses o no al mercado, tienen dimensiones económicas, pues para su realización se necesitan recursos como en cualquier otra actividad económica" (Ministerio de Cultura de Colombia, 2003, pp. 20-21).

4 No siempre resulta sencillo diferenciar la noción de bien (o mercancía) cultural respecto al concepto de servicio cultural. De hecho, los problemas derivados de su definición son motivo recurrente de debate a escala internacional, pues pese a una aparente claridad, no hay consenso con respecto a qué incluye cada categoría. En términos generales se acepta que los "productos culturales" abarcan tanto bienes como servicios. 
micas de la cultura -a la inversa del componente cada vez más cultural de la economía- impulsaron tanto a los intelectuales como a la institucionalidad cultural latinoamericana a ocuparse de identificar la oferta y la ‘demanda cultural's, así como su interacción con 'el espectador, el público y las audiencias', que durante mucho tiempo -en una visión centrada en el artista y en la obra- había quedado en segundo lugar.

De esta forma, se inicia en diversos países latinoamericanos una tradición de investigaciones fundamentales para el estudio de lo que se conoce como consumo cultural. Dos obras serán referentes obligados; primeramente, El consumo cultural en México (1993), coordinado por el antropólogo Néstor García Canclini ${ }^{6}$, y seis años más tarde la compilación El consumo cultural en América Latina (1999, 2006), de cuyo volumen dará cuenta el sociólogo chileno Guillermo Sunkel, con una variedad de perspectivas que incluye artículos de conocidos autores, como Jesús Martín-Barbero, Guillermo
Orozco, Néstor García Canclini, María Cristina Mata, entre otros. Reflexiones que van desde la aproximación a las prácticas generales en el uso del tiempo libre de los ciudadanos -cuánto de este es dedicado a ver televisión, a leer, a asistir a espectáculos, a visitar plazas o zoológicos, etc.- hasta estudios muy puntuales sobre las reacciones del público ante determinado evento o festival, la 'asistencia' a salas cinematográficas, el comportamiento de los asistentes a una determinada exposición de artes plásticas o las 'preferencias' al momento de seleccionar discos, videos y revistas. En ambos trabajos es el antropólogo Néstor García Canclini quien establece las bases conceptuales para abordar tal objeto de estudio, ya que su capítulo "El consumo cultural y su estudio en México: una propuesta teórica" (1993, pp. 15-42), que da entrada al primero de los libros mencionados, es incluido en el segundo como "El consumo cultural: una propuesta teórica" (1999, pp. 26-49).

Así tenemos que el consumo cultural como problema objeto de estu-

5 "definimos la demanda cultural como el conjunto de bienes y servicios culturales -publicaciones, actos, eventos, ferias, exposiciones- que adquieren los individuos para su uso u observación y disfrute" (Bernat, Mora y Zuluaga, 2012, p. 168).

6 Recomendamos la lectura de los siguientes libros de Néstor García Canclini: (1995). Consumidores y ciudadanos. Conflictos multiculturales de la globalización. México, D. F.: Grijalbo; (1994) (Coord.). Los nuevos espectadores. Cine, televisión y vídeo en México. México, D. F.: Coedición Consejo Nacional para la Cultura y las Artes/Instituto Mexicano de Cinematografía; y (1992) Públicos de arte y políticas culturales: un estudio del II Festival de la ciudad de México. México, D. F.: UAM-ENAH-DDF. 
dio desde la mirada de los públicos, audiencias o receptores es reciente en América Latina y el Caribe. En los últimos veinticinco años, indudablemente se ha generado un conjunto de proposiciones teóricas con sustento empírico respecto a los modos en que los públicos ven, escuchan y leen; a los usos que le dan a los bienes y servicios culturales; las maneras en que ellos relacionan esos bienes con su vida cotidiana, hasta el conjunto de teorías más importantes sobre la 'estratificación social' en el consumo cultural, a saber: 'el argumento de la homología (desarrollada por Pierre Bourdieu); el argumento de la individualización; y el argumento de la omnivoridad cultural (propuesta por Peterson y Simkus durante los años noventa)'.

En primer lugar, se encuentra el argumento de la homología, que achaca las razones implícitas para los diferentes estilos de vida y consumos culturales al factor estructural de las clases sociales y/o el estatus. En segundo lugar, el argumento de la individualización defiende que, gracias a la universalización del acceso a la cultura en las sociedades contemporáneas, las diferencias en los estilos de vida y consumos culturales están únicamente sustentadas por la voluntad del individuo. En último lugar, el argumento de la omnivoridad defiende que los gustos no son casillasestancos de propiedad exclusiva de las diferentes clases sociales, sino que la diferencia fundamental reside en cuanto del abanico de consumos culturales es capaz cada estrato de abarcar. (Herrera-Usagre, 2011, p. 143)
Pero también desde la teoría económica del consumo cultural surgirán inquietudes fundamentales referidas a los conceptos de demanda cultural y bienes culturales; 'gasto cultural de los hogares; el precio del bien o servicio cultural, el precio de bienes relacionados y el ingreso de las personas como variables explicativas; consumo cultural externo; consumo cultural doméstico; elasticidades, ingreso de la cultura', entre otras. A la par, destacan algunos autores influenciados por los estudios culturales, la semiótica, la antropología, la sociología de la cultura y la economía política; ciertos nexos críticos que van desde los análisis de los contenidos y mensajes producidos por los emisores (grandes medios de comunicación e industrias culturales) hasta lo que hoy puede referirse a un abordaje de las 'prácticas e imaginarios culturales de los latinoamericanos -lo que nos hace sentir más venezolano, mexicano, chileno, argentino o peruano'- desde una compleja y amplia visión de lo que entreteje la comunicación en este ejercicio de producción, circulación, apropiación de significados y sentidos que se liga a lo cultural.

\section{Consumo e iniquidad cultural: políticas culturales de fomento a la demanda}

El consumo no es solo reproducción de fuerzas sino también producción de sentidos: lugar de una lucha que no se agota en la posesión de los objetos, pues pasa aún más decisivamente por los usos [negritas 
añadidas] que les dan forma social y en los que se inscriben demandas y dispositivos de acción que provienen de diferentes competencias culturales (...) las luchas contra las formas de poder que atraviesan, discriminando o reprimiendo, la vida cotidiana y las luchas por la apropiación de bienes y servicios. (Martín-Barbero, 1987, p. 231)

Sin embargo, este disfrute de la cultura y activación del consumo cultural no es igual para todos. Los más pobres, los habitantes de regiones, gobernaciones, departamentos, provincias, comunas o municipios y los adultos mayores suelen estar excluidos o marginados socialmente del consumo de la oferta cultural, pública o privada, por barreras o dificultades de acceso. En ese sentido, el punto central para el diseño de unas políticas culturales genuinamente democráticas e incluyentes será: '¿Qué diferencia a los hogares que gastan en cultura de los que no lo hacen? Entre los que gastan, ¿qué diferencia a los que gastan mucho y los que gastan poco? En otras palabras, ¿qué dificulta la activación (o una mayor activación) de la demanda cultural?'.

Seguramente las respuestas son muy complejas, ya que la demanda cultural tiene diversas dimensiones, algunas cuantificables y otras no; además, la cultura y la demanda de cultura no son fáciles de medir, debido, entre otras cosas, a que el consumo cultural no implica necesariamente gastos monetarios, medibles fácilmente (Bernat, Mora y Zuluaga, 2012, p. 173), pero en la literatura especializada y en la práctica de la gestión cultural se pue- den encontrar distintos argumentos que hacen mención de circunstancias educativas, geográficas, económicas (como las elasticidades precio, precio cruzada e ingreso), demográficas (asociadas a la variable ciclo de vida, física y sensorial), psicológicas así como del entorno social, prestigio y muchas más. En algunos casos, como el acceso a Internet y en general a las tecnologías de la información y la comunicación, la brecha entre ricos y pobres es literalmente insondable.

Al problema de las oportunidades económicas -la capacidad de gasto de las personas, a partir de un cierto nivel, que determina la posibilidad de acceder a las propuestas culturales- o de la participación política se suman, en los países que integran el territorio latinoamericano, graves problemas de 'inequidad cultural'. La cultura, ciertamente, no es uno de los ítems de mayor peso en el gasto total de los hogares. Los gastos en alimentos, vivienda y otros (como salud y educación) tienen más importancia y frecuencia. Sin embargo, aún existen penosas distinciones culturales generadas por el nivel socioeconómico, educativo y etario de las personas. Conocemos, por las relaciones estadísticas, que el promedio del gasto en cultura cambia notablemente según el estrato socioeconómico y el nivel educativo. A pesar de ello, se observan también nuevas y más diferenciadas formas de consumo cultural 'entre lo culto, lo popular y lo tecnoaudiovisual', las que se pueden identificar y describir en atención a otras 
variables -tanto estructurales como subjetivas-, como las justificaciones de 'no acceso' a los bienes y servicios culturales disponibles en el espacio social. Podemos hablar -entonces- de una 'tipología de consumidores culturales para Iberoamérica' en consideración a la realización de las desigualdades socioeconómicas y diversidades culturales, según la cantidad de bienes creativos-culturales consumidos por los individuos; es decir: a) consumo cultural alto general; b) consumo cultural alto vía soporte tecnológico; c) consumo cultural medio; d) consumo cultural medio-bajo, y e) consumo cultural bajo. En el caso de los países de Latinoamérica y el Caribe, de ingreso medio y bajo, el consumo cultural es sustancialmente bajo.

Luego, parece ser cada vez más visible como acción política que las posibilidades de acceso a determinados productos culturales y de inclusión cultural -que tendrán los consumidores latinoamericanos-, se van a sostener en la aplicación de unas 'políticas culturales de fomento a la demanda'. Si definimos el 'desarrollo humano' como la capacidad de una persona para desplegar una vida que considere valiosa, es notable que el consumo cultural fuera una necesidad social inherente al impulso de las capacidades y potencialidades humanas para la realización de la vida en sus diferentes etapas. Esto supone, como metodología de intervención sociocultural, la urgencia de 'explorar, identificar y caracterizar los elementos y las relaciones que componen la estructura de consumo cultural que se está desarrollando en nuestros países, mediante la cual se asocian o disocian los destinatarios/ beneficiarios/usuarios de la oferta cultural en calidad de bienes y servicios, de acuerdo a las condiciones socioeconómicas y demográficas según el tipo de gustos, hábitos, frecuencias y tipos de dominios culturales a fin de instrumentar los resultados en el diseño de las políticas públicas culturales'.

Este interés social (el consumo cultural) se enmarca en los derechos sociales y culturales reconocidos y garantizados a toda la población latinoamericana, y ratificados en la Convención sobre la Protección y Promoción de la Diversidad de las Expresiones Culturales (20 de octubre de 2005); íntimamente interrelacionadas como expresión del conjunto de condiciones materiales y sociales, biológicas, anímicas y espirituales que definen una plena calidad de vida. El concepto de equidad cultural podría restringirse en una primera aproximación a la concepción de 'igualdad de oportunidades culturales vía consumo' que le brinda una sociedad a sus ciudadanos tanto para ejercer sus derechos civiles y políticos (libertad de expresión y reunión, libertad de elegir a sus autoridades, etc.) como sus derechos culturales y los derechos humanos en general. 


\section{El consumo cultural es más que una encuesta de opinión}

Por otra parte, contrariamente de haberse originado avances teóricos, la consolidación de los estudios sobre el consumidor cultural latinoamericano ha sido poco contundente, ya que en la gran mayoría de los países de América y el Caribe 'no existe un ordenamiento sistemático y comparativo de estadísticas culturales, y mucho menos un sistema de cuentas en el sector de la comunicación y de la cultura'. Acorde con algunas de las experiencias examinadas, hay una insuficiencia de datos y cifras sobre audiencias y públicos -fiables y completos- para un análisis significativo de las preferencias culturales y sus canales de consumo. Tenemos carencias de series históricas y antecedentes desagregados relativos a las características generales de la extensión, así como el volumen de las actividades económicas ${ }^{7}$ relacionadas con los sectores culturales y creativos nacionales que permitan un razonamiento diacrónico de un determinado período; deficiencias y dispersión de información sobre los agentes y organizaciones que participan en la cadena de valor de cada subsector de las industrias culturales, creativas y de contenidos digitales, además de su integración con los sectores productivos de cada país. En definitiva, aún no se ha desarrollado un 'Ecosistema de Información Cultural Compleja (Esicc)' -incluyendo un 'subsistema de consumo cultural'- que sirva para inventariar, organizar y sistematizar los recursos culturales (de políticas, investigación, conservación o asociativas), y así establecer un 'mapa cultural' conducente a la determinación de "índices de desarrollo cultural (IDC)' nacional, regional, estadal, municipal, parroquial y comunal; de la relación entre oferta-demanda cultural como del acceso (población atendida) a los bienes y servicios culturales.

7 Actividades económicas características de la cultura (AECC): se refiere a aquellas actividades económicas que aportan a la cultura, y que se encuentran diseminadas o comprendidas por el lado de la oferta de acuerdo a la clasificación CIIU, en diversos sectores, subsectores, ramas y actividades de la economía de un país. Ahora bien, por el lado de la demanda agregada también los bienes y servicios culturales se encuentran insertos en el consumo y en la formación bruta de capital fijo, en las exportaciones netas y las importaciones. Existen básicamente tres tipos de actividades en el sector cultural: las directas, las indirectas y los insumos. Tipo 1: corresponde a actividades directas, estrictamente ligadas con la producción del sector de la cultura. Es decir, son actividades de creación y producción original de bienes y servicios culturales. Tipo 2: incluye las actividades conexas a los procesos de producción o actividades indirectas. Son actividades que tienen que ver con el uso y la difusión de las creaciones culturales. Tipo 3: incluye los principales insumos requeridos, es decir, comprende las producciones que son demandadas por los sectores en los escenarios anteriores. 
El menoscabo de análisis con cierta prolongación en el tiempo de una manera diacrónica, sobre las ramas de actividades, los subsectores (audiovisual, fonográfico, editorial, multimedia, entre otros) y sus cadenas productivas de manera que se puedan identificar obstáculos y oportunidades para el desarrollo de sus eslabones, organizaciones y agentes ${ }^{8}$ -en particular relativo a los creadores, productores, gestores e intermediarios- se debe, en buena parte, a la exigüidad de estadísticas e indicadores que dificultan la evaluación pormenorizada del funcionamiento y actuación de los agentes que las componen. Esta parquedad de diagnósticos socioeconómicos es más apremiante, sobre todo cuando es necesario evaluar el 'impacto productivo del sector cultural-creativo a través de Cuentas Satélites de Cultura' ${ }^{9}$ (en adelante, CSC) razonado en el valor agregado por el uso de factores en los procesos de producción y distribución, y su relación con la producción nacional bruta.

A este tenor, persisten las relatividades de los términos cultura y creación que siempre han acompañado las discusiones sobre estadísticas e indicadores, y particularmente sobre consumo cultural. Se comprueba cierta ausencia de una definición explícita del sector cultural propiamente dicho dentro de las principales fuentes de información disponibles sobre variables económicas, y la mayor parte de las veces aparece de forma dispersa o mezclada con las actividades de educación e investigación, y frecuentemente con las funciones recreativas y de turismo. $\mathrm{Al}$ respecto, la Unesco está orientada a normalizar las estadísticas culturales internacionales, agregando el tan discutido concepto de 'Industrias Creati-

8 Los agentes culturales se diferencian no solo por las distintas funciones que cumplen en el proceso de producción, difusión, comercialización y consumo de los bienes y servicios culturales. Se distinguen también por la posición económica que ocupan en dicho proceso o, en otros términos, por las relaciones sociales bajo las que se inscriben en la economía cultural. En el proceso de producción, difusión, comercialización y consumo de los bienes y servicios culturales, interviene una multiplicidad de agentes, que cumplen diversas funciones. Esta diversificación de agentes es la expresión de una división social del trabajo en la producción cultural.

9 "La cuenta satélite de cultura se define como un sistema de información estadística sobre las actividades culturales que tiene como finalidad reunir en un cuadro contable coherente el conjunto de flujos económicos relacionados y establecer los vínculos entre las diferentes manifestaciones del campo cultural. La elaboración de la cuenta satélite se concibe como el medio para estructurar el conjunto de informaciones relativas al tema de la cultura. Debe dar cuenta de la importancia económica de la actividad y de la riqueza cultural de un país, incorporando indicadores monetarios y no monetarios" (DANE, Dirección de Síntesis y Cuentas Nacionales, 2007, p. 10). 
vas $^{\prime 10}$, pero solo de manera tangencial $\mathrm{y}$ haciendo eje en lo cultural, señalado en su informe denominado "Revisión del marco de referencia de las estadísticas culturales" (MEC, 2009).

En concordancia con el argumento anterior, el economista español Luis César Herrero Prieto (2002), docente de la Universidad de Valladolid, nos indica:

Probablemente el motivo de esta indefinición oficial resida en una insuficiente consolidación de los estudios económicos sobre la trascendencia del consumo y la producción cultural [cursivas añadidas]; y ésta es la razón por la que se suele acudir a definiciones ad hoc, adaptadas a la fuente de información utilizada. De esta forma, generalmente aparecen en un mismo bloque las actividades relacionadas con el ocio y la cultura, por lo que resulta obligada su consideración conjunta. (p. 147)

Para el economista cultural David Throsby (2001, p. 4) -profesor de la
Universidad Macquarie, en Sydney, Australia- la noción de cultura denota ciertas actividades emprendidas por las personas y los productos de dichas actividades, relacionadas con los aspectos intelectuales, morales y artísticos. A partir de allí se comienza a hablar de "bienes culturales", "industrias culturales" o del "sector cultural de la economía"; así como a delimitar los productos culturales como bienes y servicios que implican creatividad en su producción, incorporan un cierto grado de propiedad intelectual y transmiten un significado simbólico. En otro alcance, la concepción amplia o socio-antropológica de cultura puede ser restringida a un proceso de producción simbólica que incluye actividades generadoras de unos valores intangibles que se expresan en su 'diferencialidad' y 'unicidad' -el carácter de prototipo de la creación cultural-, y en ser bienes y servicios distintos a otros. De esta manera, la cultura pue-

10 Se consideran industrias creativas todas aquellas actividades que se generan desde la combinatoria o la intersección entre tres ámbitos disciplinarios: Arte/Cultura; Empresa/ Innovación y Ciencia/Tecnología (Unctad, 2004, p. 4). Sin embargo, existen distintas perspectivas de análisis sobre dicho concepto. John Howkins (2001) estima que se emplea para hacer referencia a todas las industrias que generan derechos de autor, patentes, marcas comerciales y diseños industriales. En otros contextos, se utiliza para referirse solo a industrias que producen contenido o industrias culturales. Así, Richard Caves (2000) entiende por industrias creativas las relacionadas con las artes, la cultura y el entretenimiento en general. También, David Throsby (2001) rescató la cultura en los debates acerca de las industrias creativas, refiriéndose a productos y servicios culturales que incluyen creatividad en su producción, abarcan algún grado de propiedad intelectual y transmiten significado simbólico. Para otros autores el término trata de diferenciarse de la visión filosófica del concepto de industria de cultura elaborado en 1947 por Max Horkheimer-Theodor Adorno desde la perspectiva de la teoría crítica de la Escuela de Frankfurt. 
de ser concebida como el campo de las producciones simbólicas, que incluye la producción artística tradicional (literatura, pintura, música, teatro y equivalentes), así como el conjunto de bienes y servicios relacionados con las denominadas "industrias culturales" o "cultura masiva" (radio, televisión, revistas, discos, conciertos, recitales, videos, cable, etc.), la "cultura popular" o "cultura folclórica" (artesanías, eventos populares del tipo ferias, "fiestas folclóricas", etc.) y de las diversas instituciones "culturales" (casas de cultura, museos, galerías, etc.) (Stolovich, Lescano y Mourelle, 2002).

Por ende, el rasgo diferencial de los estudios e investigaciones sobre los productos característicos del sector cultural es la particularidad de los bienes que son centro de análisis, es decir, los bienes y servicios culturales,

ya que todos tienen en común una triple característica: constituir un esfuerzo intelectual o creativo, representar una síntesis de belleza y poseer una carga simbólica, particular o colectiva. De esta forma, los bienes culturales pueden ser objetos tangibles (una escultura) o servicios intangibles (escuchar un concierto), pertenecer a la denominada 'alta cultura' (artes plásticas, teatro, música, literatura) o llegar hasta prototipos más cercanos al mercado como la artesanía o el diseño creativo; pero todos incluyen un elemento artístico y una vocación estética. Además, estos bienes suelen implicar alguna propiedad intelectual y muchos de ellos, sobre todo los pertenecientes al patrimonio cultural, provocar externalidades difícilmente cuantificables en el mercado. Esta singularidad de la naturaleza de los bienes y servicios culturales es lo que les diferencia intrínsecamente del resto de bienes ordinarios de una economía e interpone una primera barrera a la aplicación del análisis económico. La principal dificultad consiste en la confección y aplicabilidad de una teoría del valor consistente, no sólo porque en los bienes culturales, a diferencia del resto, pueden distinguirse dos acepciones de valor, indisociables pero distintas (valor cultural y valor económico) sino porque también resulta complicado su computo. (Herrero, 2009, pp. 37-38)

\section{Qué significa consumir cultura. La particularidad del sector y campo cultural}

Es posible definir la particularidad del consumo cultural como el conjunto de procesos de apropiación y usos de productos en los que el valor simbólico prevalece sobre los valores de uso y de cambio, o donde al menos estos últimos se configuran subordinados a la dimensión simbólica [negritas añadidas]. Esta definición permite incluir en el ámbito peculiar del consumo cultural no solo los bienes con mayor autonomía: el conocimiento universitario, las artes que circulan en museos, salas de concierto y teatros. También abarca aquellos productos muy condicionados por sus implicancias mercantiles (los programas de televisión) o por la dependencia de un sistema religioso (las artesanías y las danzas indígenas), pero cuya elaboración y cuyo consumo requieren un entrenamiento prolongado en 
estructuras simbólicas de relativa independencia (García Canclini, 1999, pp. 42-43).

Definitivamente, la cultura es un proceso social de producción simbólica, que surge del trabajo creativo. La creación cultural y su difusión social constituyen momentos que encierran la creación de signos, la producción de soportes materiales de esos signos o de 'presentaciones en vivo' de estos, su difusión entre los receptores/consumidores y su atesoramiento. Pero, a menudo, la cultura es considerada en un sentido mucho más restrictivo, como el conjunto de producciones o de productos (bienes y servicios) culturales: obras de todo tipo, musicales, teatrales, cinematográficas, televisivas, etcétera. De acuerdo con ello, lo que se denomina el sector cultural alcanza un conjunto de ámbitos de ramas económicas e industriales muy amplio. Se incluyen actividades de creación, producción, manufactura y distribución de bienes y servicios relacionadas con las siguientes áreas y subáreas: el patrimonio; artísticas (de la creación) que abarcan la literatura, las artes visuales, la danza, el teatro, la música; las artes del espectáculo; los medios de comunicación: diarios y revistas, la televisión abierta y por suscripción, la radio, y las artes audiovisuales. En este sentido, la cultura toma una dimensión profesional y se ve implicada de inmediato con aspectos económicos, gerenciales y administrativos. El Convenio Andrés Bello (CAB), en los documentos que ha publicado a propósito de la elabora- ción metodológica de Cuentas Satélites de Cultura (CSC), define al 'campo cultural' como un 'conjunto de actividades humanas y productos cuya razón de ser consiste en crear, expresar, interpretar, conservar y transmitir contenidos simbólicos'.

Una vez definido el campo cultural se hace la distinción entre 'productos característicos' y 'productos conexos'. Entre los característicos se consideran los productos típicos de la cultura: libros, obras de arte, películas, conciertos, etc. En los conexos se incluyen los bienes y servicios que hacen parte de los gastos culturales, tales como videograbadoras, cámaras fotográficas, televisores, radios, etc. En una primera aproximación podríamos decir entonces que el sector cultural está constituido por:

a) el arte, en sus diversas manifestaciones (música, teatro, plástica, artesanía, etc.), incluyendo el espectáculo artístico en vivo, el patrimonio cultural y su conservación (museos, etc.); se trata de las "bellas artes" y de otras artes excluidas de los conceptos restrictivos de cultura;

b) las denominadas industrias culturales, $\mathrm{y}$

c) los medios masivos de comunicación (radio, televisión, prensa, etc.) que establecen un nexo, a escala de masas, entre la producción cultural y los receptores/consumidores de cultura, creando una red por la que circulan los bienes culturales. 
Teniendo en cuenta esta clasificación, consumir cultura

significa entonces relacionarnos con una oferta ligada al entretenimiento (fiestas, celebraciones), a la información (periódicos, Internet, revistas, diarios) y a experiencias estéticas (artes visuales, conciertos, teatro, etc.), pero, al mismo tiempo, satisfacemos otras necesidades como la identificación grupal, regional, nacional o multinacional, nos distinguimos socialmente (y simbólicamente), logramos sociabilidad con otros por medio de ritos (expresión) y apropiación de espacios públicos (prácticas) y, a la vez, participamos (en distintas formas culturales) en el mundo. (Güell, Peters y Morales, 2010, p. 48)

Pero, ¿cuál es la particularidad que tendrán los productos culturales? Asumiendo que el sector cultural y aquellas actividades económicas vinculadas a la producción cultural están conformados por una serie de bienes y servicios de distinto tipo, el 'valor simbólico', es decir, la manifestación simbólica de una 'función cultural' asociado a estos (valores, creencias, normas, símbolos expresivos) es determinante. Aparte de poseer un importante componente público, los bienes y servicios culturales tienen otras características que los distinguen del común de los productos de mercado ${ }^{11}$. Su distinción no reside, rigurosamente hablando, solamente en características de la naturaleza de la cultura considerada como bien económico, se asienta también en rasgos propios del proceso productivo en el sector cultural. Un aspecto básico es que estamos hablando de bienes y servicios que intentan satisfacer un tipo de necesidad específica: la cultural. Esta es la única característica excluyente con respecto a otro tipo de productos, y tiene la particularidad de ser definida por la interacción de la demanda y la oferta (Consejo Nacional de la Cultura y las Artes de Chile, 2003, p. 21), contribuyendo a determinar la originalidad de estos. Estamos en presencia de unos productos de características singulares, que no cuentan con una definición concisa pero que además no tienen un sentido propio, intrínseco, sino que el consumidor cultural le otorga un significado particular, dependiendo de lo que este represente para él. Un elemento clave del consumo cultural es el efecto de la experiencia y el entrenamiento en beneficio de uno mismo al consumir bienes culturales-creativos; este efecto es llamado 'adicción racional'.

11 Según el economista cultural Frey (2000, pp. 15-16) las características que hacen del arte y la cultura un "bien público" que produce "efectos externos positivos", cuyos beneficios no se agotan en las personas que los demandan y ofrecen como bien privado a través del libre mercado, son: valor de existencia, valor de prestigio, valor de opción o elección, valor de educación y un valor de legado. 
Con algunas excepciones, como la música que se escucha en la radio, los productos de las industrias de contenido cultural y creativo son 'bienes de experiencia'. Para complicar más la situación, los bienes culturales-creativos poseen -además de su valor funcional- un valor simbólico y emocional que es distinto para cada individuo o grupo de individuos; que son irremplazables, ya que responden a necesidades que no pueden ser cubiertas por ningún otro bien. Otro elemento o particularidad que dificulta su examen económico está representado por su comportamiento dentro del mercado, pues se comportan de manera diferente a los demás bienes, dado que su permanencia o durabilidad en el mercado no puede determinarse a priori.

\section{El consumo cultural como objeto de estudio en el espacio geográfico cultural latinoamericano}

[Se puede proponer], que el consumo cultural se refiere a los distintos tipos de apropiación de aquellos bienes cuyo principal valor percibido es el simbólico, que es producido en circuitos relativamente diferenciados $y$ que requiere de ciertos conocimientos especializados para su apropiación y uso [negritas añadidas] (Güell, Peters y Morales, 2010, p. 48).

Nos hallamos frente a un campo del conocimiento, de muy reciente impulso, fecundo en la diversidad de vetas que se han explorado y en las metodologías puestas en práctica. Los estímulos para el desarrollo de exploraciones, radiografías y mapeos (encuestas, sondeos, informes, etc.) sobre el consumidor cultural latinoamericano han provenido fundamentalmente de tres ámbitos: instituciones gubernamentales de cultura (ministerios de cultura y consejos de artes), espacios académicos (universidades e institutos de investigación) e industrias culturales, fundamentalmente de agencias de mercadeo y publicidad. En el marco de formulación de políticas públicas culturales, los países latinoamericanos están procurando definir el perfil de la demanda cultural con la aplicación de encuestas nacionales de consumo cultural:

Fuentes de información más completa y reciente, tanto por los aspectos que se incluyen y se miden como por el tamaño de la muestra; no solo incluye datos sobre hábitos de consumo sino también sobre características socioeconómicas, gastos de consumo e ingresos de las personas. (Bernat, Mora y Zuluaga, 2012, p. 174)

No obstante, tal como señala la antropóloga e investigadora mexicana Ana Rosas Mantecón (2002):

A pesar de los importantes avances realizados en los últimos años en términos de construcción teórica y de líneas de investigación, el estudio del consumo cultural se sigue planteando como un doble desafío: teórico [cursivas añadidas], porque no se ha construido aún un enfoque transversal capaz de describir y explicar los procesos de consumo cultural, que son regulados 
por racionalidades diversas (económicas, políticas, simbólicas) y que se encuentran íntimamente vinculados a una gama amplia de prácticas y fenómenos sociales que los atraviesan y condicionan; metodológico [cursivas añadidas] también, puesto que no se han evaluado suficientemente los alcances y límites de la aplicación de técnicas cualitativas (como la entrevista individual y grupal, la historia de vida y el relato, el análisis del discurso, la observación participante, etc.) y cuantitativas (la encuesta). (p. 9)

Haciendo nuestras las reflexiones de Ana Rosas Mantecón (2002, p. 8) sobre este tema, podemos señalar que en el espacio geográfico cultural iberoamericano, no significa que se trate de una línea de investigación inédita o de una acción política cultural por construir; por el contrario, las publicaciones sobre el consumo cultural tienen una historia propia en el pensamiento sociocultural latinoamericano, la cual nos permite orientar nuevos desafíos investigativos. Lo que sí es cierto es que han derivado en intentos aislados sin continuidad y sin el debido reconocimiento por la institucionalidad pública cultural -sobre todo en la obtención de recursos financieros por los costos de las encuestas de carácter nacional- para realizar este tipo de estudios, cuya naturaleza es esencialmente interdisciplinaria (sociología de la cultura, antropología social, semiótica, estética de la recepción, estadística, comunicación, psicología social, etc.). Es capital acentuar que no todos los países latinoamericanos han aportado información sobre el tema y esta baja tasa de respuesta no debe ser considerada como una señal de falta de interés, sino más bien, deberse al hecho de que el contenido abordado constituye un área de actividad muy específica, con un marcado carácter de frontera y una incorporación tardía de nuestras instituciones gubernamentales de cultura (ministerios de cultura y consejos de artes) y espacios académicos.

En el ámbito latinoamericano destacan las investigaciones realizadas, entre otros, por Óscar Landi, Patricia Terrero, María Cristina Mata, Nora Mazzioti, Ana Wortman, Rubens Bayardo, Florencia Saintout, Natalia Ferrante, Luis Alberto Quevedo, Roberto Bacman, Lelio Alberto Mármora y Marcelo Padilla (Argentina); Marcelo Guardia Crespo (Bolivia); Antonio Arantes, Silvana Rubino, Sergio Miceli, Nilda Jacks y Ana Carolina Escosteguz (Brasil); Guillermo Sunkel, José Joaquín Brunner, Carlos Catalán, Valerio Fuenzalida Fernández, Alicia Barrios, Pablo Torche, Cristian Antoine, Modesto Gayo, María Luisa Méndez, Pedro Güell Villanueva, Tomás Peters Núñez y Rommy Morales Olivares (Chile); Jesús Martín-Barbero, María Patricia Téllez, Sonia Muñoz, Helena Useche Aldana y Germán Rey (Colombia); Ana Cecilia Montilla y Carlos Ávalos (Costa Rica); Cecilia Linares, Yisel Rivero, Pedro E. Moras y Yosleidy Mendoza (Cuba); Amparo Marroquín Parducci (El Salvador); Víctor Fernández Blanco, Juan Prieto Rodríguez, Cristina Muñiz Artime, Rubén Gutiérrez del Castillo, Jordi 
López Sintas, Ercilia García Álvarez, Luis Enrique Alonso, Aniano Hernández Guerra, Manuel Cuadrado y José Luis Piñuel Raigada (España); Néstor García Canclini, Ana Rosas Mantecón, Mabel Piccini, Eduardo Nivón Bolán, Guillermo Orozco, María Rebeca Padilla de la Torre, Graciela Schmilchuk, José Carlos Lozano, Lorena Frankenberg y José Antonio Meyer Rodríguez (México); Hugo Achugar, Sandra Rapetti, Susana Dominzain, José Mourelle, Luis Stolovich, Graciela Lescano, Rosario Radakovich, Debora Duarte, Luisina Castelli Rodríguez y Rosario Sánchez Vilela (Uruguay); Marcelino Bisbal, Pasquale Nicodemo, Jesús María Aguirre, Tulio Hernández, Carlos Guzmán Cárdenas, Emilia Bermúdez, Natalia Sánchez, Gustavo Hernández Díaz y Carlos Delgado Flores (Venezuela).
Para el año 2014 se incorporó la República Dominicana a la lista de países de América Latina y el Caribe que realizan estudios de consumo cultural para la evaluación de sus políticas culturales, con los resultados de su primera Encuesta Nacional de Consumo Cultural (en adelante, ENCC-RD 2014). La ENCC-RD 2014 fue realizada por un equipo multidisciplinario del Ministerio de Cultura y el Banco Central de la República Dominicana como Módulo de la Encuesta Nacional de Fuerza de Trabajo (en adelante, ENFT) -aplicada de manera semestral en los meses de abril y octubre de cada año-para dar apoyo al proyecto Cuenta Satélite de Cultura de la República Dominicana (en adelante, CSC-RD). De allí que la República Dominicana, junto a otras naciones como Argentina ${ }^{12}$,

12 Consúltese: Secretaría de Medios de Comunicación de la Jefatura de Gabinete de Ministros de la Presidencia de la Nación (2005). Sistema Nacional de Consumos CulturalesSNCC. Agosto 2005. Uno. Buenos Aires. Argentina. Sistema de Información Cultural de la Argentina (SInCA); Secretaría de Medios de Comunicación de la Jefatura de Gabinete de Ministros de la Presidencia de la Nación (2006). Sistema Nacional de Consumos CulturalesSNCC. Marzo 2006. Dos. Buenos Aires. Argentina. Sistema de Información Cultural de la Argentina (SInCA); Secretaría de Medios de Comunicación de la Jefatura de Gabinete de Ministros de la Presidencia de la Nación (2006). Sistema Nacional de Consumos Culturales SNCC. Noviembre 2006. Tres. Buenos Aires. Argentina. Sistema de Información Cultural de la Argentina (SInCA); Secretaría de Medios de Comunicación de la Jefatura de Gabinete de Ministros de la Presidencia de la Nación (2008). Sistema Nacional de Consumos Culturales SNCC. Marzo 2008. Cuatro. Buenos Aires. Argentina. Sistema de Información Cultural de la Argentina (SInCA); Dirección Nacional de Industrias Culturales (2013). Encuesta Nacional de Consumos Culturales y Entorno Digital. Argentina. Sistema de Información Cultural de la Argentina (SInCA).

13 Se recomienda consultar y evaluar los estudios del Instituto Nacional de Estadísticas correspondientes a los años: (2009) Enfoques estadísticos. Encuesta exploratoria de uso de tiempo libre en el Gran Santiago. Boletín Informativo del Instituto Nacional de Estadísticas. Santiago de Chile. Consejo Nacional de la Cultura y las Artes. INE Chile. Mayo; (2008) 
Chile $^{13}$, Colombia ${ }^{14}$, Costa Rica ${ }^{15}, \mathrm{Cu}-$ $\mathrm{ba}^{16}$, México ${ }^{17}$, Uruguay ${ }^{18}$ y Venezue-
$1 \mathrm{a}^{19}$ se incorpore en la construcción institucional de una mirada prospec-

Enfoques estadísticos. Cultura y tiempo libre. Boletín Informativo del Instituto Nacional de Estadísticas. Santiago de Chile. Consejo Nacional de la Cultura y las Artes. INE Chile. Enero; (2006) Enfoques estadísticos. Los chilenos y la cultura. Boletín Informativo del Instituto Nacional de Estadísticas. Santiago de Chile. Consejo Nacional de la Cultura y las Artes. INE Chile; (2006a) Enfoques estadísticos. Los chilenos y la cultura. Boletín Informativo del Instituto Nacional de Estadísticas. Santiago, Chile. Diciembre. Consejo Nacional de la Cultura y las Artes. INE Chile. Véase Gobierno de Chile (2007) Encuesta de consumo cultural 2004-2005. Valparaíso, Chile. Programa Encuesta de Consumo Cultural. Consejo Nacional de la Cultura y las Artes. Departamento de Planificación y Estudios. Unidad de Estudios y Documentación; Consejo Nacional de la Cultura y las Artes (2009) Segunda ENPCC. Encuesta Nacional de Participación y Consumo Cultural. Santiago, Chile. Consejo Nacional de la Cultura y las Artes. Ediciones Cultura; Consejo Nacional de la Cultura y las Artes (2009). Encuesta Nacional de Participación y Consumo Cultural. Síntesis descriptiva. Estudios. Santiago de Chile. Consejo Nacional de la Cultura y las Artes. Unidad de Estudios y Documentación, Departamento de Planificación y Estudios; Consejo Nacional de la Cultura y las Artes (2013) Encuesta Nacional de Participación y Consumo Cultural. Análisis descriptivo. ENPCC 2012. Estudios. Santiago, Chile. Consejo Nacional de la Cultura y las Artes. Publicaciones Cultura; Consejo Nacional de la Cultura y las Artes (2014) Análisis y levantamiento cualitativo: Participación y prácticas de consumo cultural. Informe final. Santiago de Chile. Sección Observatorio Cultural del Departamento de Estudios del Consejo Nacional de la Cultura y las Artes.

14 Consultar y evaluar: Departamento Administrativo Nacional de Estadísticas (2008) Encuesta de Consumo Cultural 2007. Informe de Resultados. Bogotá, Colombia. Dirección de Regulación, Planeación, Estandarización y Normalización (Dirpen), abril; Departamento Administrativo Nacional de Estadísticas (2009) Encuesta de Consumo Cultural 2008. Informe de Resultados. Bogotá, Colombia. Dirección de Regulación, Planeación, Estandarización y Normalización (Dirpen), marzo; Departamento Administrativo Nacional de Estadísticas (2010) Encuesta de Consumo Cultural 2010. Informe de Resultados. Bogotá, Colombia. Dirección de Regulación, Planeación, Estandarización y Normalización (Dirpen). Todos los datos son expandidos con proyecciones de población, con base en los resultados del Censo de Población 2005; Departamento Administrativo Nacional de Estadísticas (2013) Encuesta de Consumo Cultural 2012. Informe de Resultados. Bogotá, Colombia. Dirección de Regulación, Planeación, Estandarización y Normalización (Dirpen), abril. Todos los datos son expandidos con proyecciones de población, con base en los resultados del Censo de Población 2005; Departamento Administrativo Nacional de Estadísticas (2014) Encuesta de Consumo Cultural 2014. Informe de Resultados. Bogotá, Colombia. Dirección de Regulación, Planeación, Estandarización y Normalización (Dirpen), diciembre. Periodo de recolección agosto-septiembre 2014.

15 Consultar Montilla, A. C. y Ávalos, C. (2012). Análisis de la encuesta de prácticas y hábitos culturales de Costa Rica 2010-2011. San José de Costa Rica: Ministerio de Cultura y Juventud. 
tiva latinoamericana sobre los cambios en la producción, circulación y consumo de nuestros productos culturales, proporcionando información estadística e índices básicos generales sobre hábitos, prácticas/asistencia y gastos realizados por los hogares en la adquisición de bienes creativos-

16 Véanse Linares, C., Rivero, Y., y Moras, P. E. (2008). Participación y consumo cultural en Cuba. La Habana: Instituto Cubano de Investigación Cultural Juan Marinello; Linares, C., Rivero, Y., Moras, P. E., y Mendoza, Y. (2010). El consumo cultural y sus prácticas en Cuba. La Habana: Instituto Cubano de Investigación Cultural Juan Marinello.

17 Consejo Nacional para la Cultura y las Artes (2004). Encuesta nacional de prácticas y consumo culturales. México, D. F.: Conaculta; Consejo Nacional para la Cultura y las Artes (2010). Encuesta nacional de hábitos, prácticas y consumo culturales. México, D. F.: Conaculta; Goldin, D. (Ed.) (2006). Encuesta nacional de lectura. Informes y evaluaciones. México, D. F.: UNAM-Conaculta; Consejo Nacional para la Cultura y las Artes (2009). Encuesta a públicos de bibliotecas 2008. Informe de resultados. México, D. F.: Conaculta; Consejo Nacional para la Cultura y las Artes (2008). Encuesta a públicos de librerías 2007-2008. Informe de resultados. México, D. F.: Conaculta; Consejo Nacional para la Cultura y las Artes (2009). Encuesta a públicos de la Orquesta Sinfónica Nacional 2009. Informe de resultados. México, D. F.: Conaculta; Consejo Nacional para la Cultura y las Artes (2009). Encuesta a públicos de teatros 2009. Informe de resultados. México, D.F.: Conaculta; Consejo Nacional para la Cultura y las Artes (2009). Encuesta a públicos de museos 2008-2009. Informe de resultados. México, D. F.: Conaculta; Instituto Nacional de Estadística y Geografía (INEGI) (2012). Encuesta nacional de consumo cultural de México. México, D. F.: Instituto Nacional de Estadística y Geografía/Conaculta.

18 Achugar, H., Rapetti, S., Dominzain, S., y Radakovich, R. (2003). Imaginarios y consumo cultural. Primer informe sobre consumo y comportamiento cultural. Uruguay 2002. Montevideo: Universidad de la República Uruguay/CEIL/Ediciones Trilce; Dominzain, S., Rapetti, S., y Radakovich, R. (2009). Imaginarios y consumo cultural. Segundo informe nacional sobre consumo y comportamiento cultural. Uruguay 2009. Montevideo: Universidad de la República Uruguay/Ministerio de Educación y Cultura; Dominzain, S., Radakovich, R., Duarte, D., y Castelli Rodríguez, L. (2014). Imaginarios y consumo cultural. Tercer informe nacional sobre consumo y comportamiento cultural. Uruguay 2014. Montevideo: Universidad de la República Uruguay/Ministerio de Educación y Cultura.

19 Consúltese: Bisbal, M., Nicodemo, P., Aguirre, J. M., Guzmán Cárdenas, C. E., Pellegrino, F., y Pilato, E. (1998). El consumo cultural del venezolano. Caracas: Fundación Centro Gumilla y Consejo Nacional de la Cultura (Conac); Bisbal, M., y Nicodemo, P. (2007). Nuevos medios para ¿nuevas prácticas? Revista Comunicación. Estudios Venezolanos de Comunicación, 138, pp. 38-54. Caracas: Centro Gumilla; Bisbal, M., Nicodemo, P. (1997). El valor de la cultura. El consumo cultural del venezolano. En Demoscopio Venezuela. Tendencias y perspectivas 1996-1997, pp. 43-48. Caracas: COSAR Grupo Comunicacional; Bisbal, M., y Nicodemo, P. (2010).El consumo del venezolano en la comunicación y la cultura. Internet avanza pero la TV no cede. Revista SIC, 725. Dosier, 211-222. 
culturales, disponibles en República Dominicana, con el fin de propiciar la elaboración de la CSC-RD.

Puede advertirse, por consiguiente, que en los estudios de consumo y demanda cultural el cotejo de las prácticas, hábitos, preferencias y comportamientos del consumidor cultural, ligados a valores simbólicos, entre cuyas características destaca la inmaterialidad o la "espiritualidad" como atractivos dominantes, resulta estratégico para 'reconocer y reafirmar la diversidad de expresiones culturales'. Además, para conocer las 'imágenes e imaginarios en virtud de las cuales una sociedad se reproduce', y en particular, para la aplicación de políticas y medidas tomando en cuenta los perfiles de identidades a través de la cual se reconoce a 'sí misma' y cambia, a fin de instrumentar este discernimiento para una gestión cultural democrática. Requerimos de precisiones muy afinadas que nos digan 'cómo se está moviendo el ciudadano en orden a lo simbólico que no es más que su producción y consumo cultural'. No obstante, la primera dificultad para abordar el tema ha sido la discusión sobre lo que en definitiva se podrá entender como "consumo" y, por supuesto, por "consumo cultural". En general, siempre ha estado asociado a gastos suntuarios, escenarios del control económico o consumismo. Los economistas, de manera bastante compleja, lo explican por relaciones entre precios y salarios, inflación e índices de precios al consumidor, leyes de expansión y con- tracción de los mercados; las ciencias sociales se han interesado por los factores cualitativos que determinan las interacciones sociales. En términos generales, se puede plantear, en la línea propuesta por la Corporación Centros de Estudios, Investigación y Comunicación Social (Ceicos) (1998), que concebiremos al consumo cultural como:

La serie de actividades y actitudes individuales y grupales, públicas y privadas de los ciudadanos, en las cuales compran, usan, asisten, ven, escuchan, sienten, disfrutan e interactúan con la diversa gama de la oferta cultural. Este se refiere al fenómeno causado por el conjunto de personas, parejas, familias, grupos homogéneos y heterogéneos, de legos, cultos y aficionados en el terreno cultural, y hacen parte del llamado capital cultural y humano de la sociedad y la economía modernas. (p. 94)

$\mathrm{Y}$ entenderemos por consumidor cultural:

La persona o las personas que establecen relaciones de uso, valor, apropiación o apreciación con cualquier área o elemento de la oferta cultural. Este es comúnmente el comprador, espectador, asistente o usufructuante del elemento y/o acto cultural, de los bienes materiales y simbólicos. Este está mediado por una relación mercantil, benéfica, altruista o de goce, con el panorama cultural del entorno público o privado. Puede ser un aficionado, un artista, un especialista, un estudiante, un lego, un individuo solitario, una pareja, una familia o un grupo ocasional o intencionalmente interesado en la actividad cultural. (Ceicos, 1998, p. 94) 
Lo cierto del caso es que, acercándonos a una noción proveniente de distintas disciplinas, podríamos definirlo como un acto donde las clases y grupos compiten por la apropiación del producto social, que distingue simbólicamente, integra y comunica, objetiva los deseos y ritualiza su satisfacción (García Canclini, 1999). Desde esta perspectiva, la determinación de las características del consumo cultural del espacio geográfico cultural de América Latina y el Caribe exige la superación de las concepciones unilaterales y el reconocimiento de la función del consumo en la vida social. Por ende, es pertinente tener un conocimiento cabal acerca de:

1. La segmentación de los públicos y los cambios en las pautas de consumo cultural.

2. La recomposición de los espacios culturales ante la hibridación de los múltiples procesos simbólicos que se cruzan y fecundan mutuamente.

\section{El interés de los estudios de consumo cultural en Latinoamérica}

El análisis del consumo de bienes y servicios culturales, así como la reflexión en torno a los usos del tiempo libre, han adquirido un inusitado protagonismo en los últimos veinticinco años en la reflexión de las ciencias sociales, las teorías de la comunicación, el marketing y, más en general, en la formulación de políticas públicas culturales. Dicho interés se ha ido asentando progresivamente en el espacio geográfico cultural iberoamericano, donde son especialmente relevantes los casos de España, México, Colombia, Chile y Argentina, y en menor medida Uruguay, Costa Rica, Cuba y Venezuela. Generalmente se entiende por consumo cultural el acceso a bienes y servicios tales como libros, discos y fonogramas, funciones de cine, conciertos, representaciones teatrales y danza, diarios, revistas, televisión, etc.; es decir, los productos originados por el conjunto de ámbitos productivos que conforman el sector cultural. Se habla de públicos de museos o de teatro, espectadores del cine, audiencias de medios radioeléctricos, receptores audiovisuales, abonados de televisión por suscripción, internautas del ciberespacio, comunidades de redes sociales, net-compulsivos del comercio electrónico, pero en realidad solo hay 'consumidores híbridos', aunque cabe preguntarse -como apunta el Consejo Nacional de la Cultura y las Artes en la Encuesta Nacional de Participación y Consumo Cultural de Chile del año 2012 (2013, p. 18), por la presencia de consumidores culturales omnívoros en nuestro espacio geográfico e indagar respecto a cuáles son los segmentos sociales más cercanos al 'omnivorismo cultural', que se destacarían por consumir sin distinción alta cultura y cultura popular, asociadas respectivamente a las clases dominantes y dominadas, es decir, el consumidor omnívoro va al cine y a la ópera, escucha música clásica, 
folclórica y romántica. De igual manera, investigar la evolución de la asociación entre 'prestigio y consumo cultural' y los cambios que ha experimentado esta relación como consecuencia del desdibujado incentivo social -entendido como prestigio académico-que movía las prácticas culturales aspiracionales.

Un enfoque transversal de consumos, así como el estudio de sus articulaciones entre distintos hábitos $\mathrm{y}$ prácticas culturales revelaría los perfiles y requerimientos complejos de los 'destinatarios/beneficiarios/usuarios' de cada producto. Estas demandas de conocimiento hacen necesario examinar en forma conjunta-con un enfoque descriptivo antes que de exhaustividad-, algunos estudios y encuestas de vocación nacionales realizados por instituciones gubernamentales de cultura en América Latina, con el propósito de tener una visión del consumo cultural en América Latina. Es importante destacar dos estudios de cobertura iberoamericana.

El primero refiere a la investigación titulada La industria cinematográfica y su consumo en los países de Iberoamérica ${ }^{20}$ (2004) y coordinada por Carlos Enrique Guzmán Cárdenas en el marco del convenio internacional Ininco-CAACI. El objetivo general correspondió a una investigación descriptiva, de tipo analítico-documental, con un enfoque diacrónico, para indagar comparativamente cómo se han modificado los elementos y las

20 Guzmán Cárdenas, C. E. (2004). La industria cinematográfica y su consumo en los países de Iberoamérica. Caracas: Venezuela. Investigación realizada para Conferencia de Autoridades Audiovisuales y Cinematográficas de Iberoamérica (CAACI), con la colaboración del Centro Nacional Autónomo de Cinematografía (CNAC) de Venezuela. Equipo de investigación: Yolanda Quintero Aguilar y Alfredo Caldera. Del mismo autor, Guzmán Cárdenas, C. E. (2006). La industria cinematográfica y su consumo en los países de Iberoamérica. Un análisis comparativo diacrónico. En Anuario Ininco/Investigaciones de la Comunicación. Caracas: Universidad Central de Venezuela, Instituto de Investigaciones de la Comunicación, Facultad de Humanidades y Educación. Volumen 18, N.1, pp. 57-118; (2001) La demanda, la pobreza, la inversión y el consumo cultural en Venezuela. Revista Comunicación. Estudios Venezolanos de Comunicación, 113, 12-19. Caracas: Centro Gumilla; (1998) La ciudad como objeto de consumo cultural. En Bisbal, M., Nicodemo, P., Aguirre, J. M., Guzmán Cárdenas, C. E., Pellegrino, F. y Pilato, E. El consumo cultural del venezolano, pp. 136-169. Caracas: Fundación Centro Gumilla y Consejo Nacional de la Cultura (Conac); (1996) La demanda del 'nosotros': descubriendo la ciudad como acontecimiento de consumo cultural. En Bisbal, M., y Nicodemo, P. (coordinación). Nuevas fronteras. Medios, comunicación y poder, pp. 115-130. Caracas: Fundación Carlos Eduardo Frías/Universidad Central de Venezuela; (1995) Asimetrías de la urdimbre cultural venezolana. Políticas culturales y públicos. Revista Comunicación. Estudios Venezolanos de Comunicación, 92, 5-21. Caracas: Centro Gumilla; (1995a) Políticas culturales y públicos. En Museos Ahora, 3, 27-40. Caracas: Dirección General Sectorial de Museos del Consejo Nacional de la Cultura. Museos y Público. 
relaciones que componen la estructura del consumo cultural cinematográfico que se está desarrollando en los países miembros de la Conferencia de Autoridades Audiovisuales y Cinematográficas de Iberoamérica (CAACI), en el periodo de 1989-2005, en atención a los resultados de las más importantes y rigurosas investigaciones académicas y estudios de opinión especializados, a fin de instrumentar los resultados en el diseño de políticas culturales.

En tal sentido, la intencionalidad analítica de la investigación, en su enfoque diacrónico, fue el de proporcionar una visión del "estado del arte" sobre las producciones académicas y estudios de mercado especializados que compartían el común denominador de explorar las dimensiones de consumo audiovisual del espectador cinematográfico (cinéfilo).

El segundo estudio es la Encuesta latinoamericana de hábitos y prácticas culturales (2013), auspiciada por la Organización de Estados Iberoamericanos (OEI), con el apoyo técnico de la Corporación Latinobarómetro.

Se indican los hábitos de consumo cultural en una gran variedad de dimensiones: lectura de periódicos $\mathrm{y}$ libros, televisión, radio, cine, teatro, video, música, la utilización de computadoras, el acceso al correo electrónico, Internet y redes sociales, las visitas a lugares del patrimonio cultural, la celebración de eventos culturales, la asistencia a conciertos y, en general, el acceso a los bienes y servicios culturales y su participación en ellos. Al final, se plantean dos preguntas de interés: ¿cómo valora usted la oferta cultural realizada en su país en los diez años anteriores? y ¿qué expectativas tiene sobre la oferta cultural en los próximos diez años? La investigación se elaboró y desarrolló a lo largo de 2013, y el informe final se preparó en el primer semestre de 2014.

En términos generales, los hallazgos principales de dicha encuesta para el espacio geográfico cultural latinoamericano -se consultaron 16 países de la región; Panamá y República Dominicana no fueron incluidos en la última fase del estudio por incongruencias en los datos reportados- señalan como principales conclusiones (OEI, 2014, pp. 159-165) que:

- Los porcentajes de los latinoamericanos encuestados que 'no han accedido "nunca o casi nunca»' a las actividades mencionadas en la encuesta son: $68 \%$ a conciertos, recitales, etc.; $67 \%$ al teatro; $65 \%$ al cine; $62 \%$ a patrimonio material; $60 \%$ celebración comunitaria; $55 \%$ correo electrónico/ Internet; $53 \%$ computadora; $45 \%$ lectura por profesión/estudio; $42 \%$ lectura por diversos motivos; $40 \%$ video; y música grabada $32 \%$.

- Mientras en Europa nos encontramos con un $32 \%$ de ciudadanos que no han leído un libro en los últimos doce meses, en América Latina este porcentaje es de $42 \%$. La 'no asistencia al teatro' en Europa alcanza al 72 
$\%$ de la población y en América Latina al $67 \%$, y la 'no asistencia a conciertos' se sitúa en un $65 \%$ en Europa y un $68 \%$ en América Latina.

- Las actividades culturales más frecuentadas son la audición de música, el video y la lectura. Son prácticas que pueden realizarse en múltiples contextos (hogar, desplazamientos), de forma individual o en grupo, con total libertad de horarios. Y por lo general, tienen un cierto carácter gratuito, en el sentido de que no es necesario el pago de una entrada cada vez que uno realiza dicha práctica, aunque en algunos casos sí pueda suponer un cierto desembolso inicial.

- En el extremo contrario se sitúa la asistencia a conciertos, al teatro o al cine. Estas prácticas requieren la asistencia a un evento, en lugar y horas determinados, $y$, por lo general, el pago de una entrada.

- La casi totalidad de la población latinoamericana oye radio y ve la televisión. Son, sin duda, las prácticas más extendidas. Las diferencias de género, edad, nivel de estudio y nivel socioeconómico no son en este caso especialmente significativas, aunque existen. El número de horas semanales destinadas a estas prácticas pudiera parecer elevado, pero está en línea con lo que sucede en otras regiones del mundo.

- En una zona intermedia se sitúan dos prácticas relacionadas con las nuevas tecnologías de la comunicación: el uso del correo electrónico y la Internet.
- Las variables que mayor peso tienen, con gran diferencia sobre las demás, son el nivel socioeconómico $\mathrm{y}$, sobre todo, el nivel educativo. Hay dos actividades que están muy extendidas entre los ciudadanos: escuchar radio y ver televisión. Las diferencias de género, edad, nivel de estudios y nivel socioeconómico se reducen en estos casos al mínimo.

- La edad marca también una cierta diferencia, más acentuada como era de esperar en lo que se refiere al uso de la computadora e Internet. Sin embargo, si nos referimos a la lectura como placer, la asistencia al teatro o la visita a lugares patrimoniales, la diferencia de frecuencia entre los jóvenes y los mayores es significativamente menor que en otras prácticas culturales.

- La variable género marca diferencias de frecuencia mucho menores que las otras tres variables en casi todas las prácticas estudiadas. Las diferencias por razones de género llegan a ser prácticamente inexistentes en lo que se refiere a lectura por placer, a celebraciones comunitarias y a visitas a lugares patrimoniales.

\section{Consideraciones finales}

Es evidente que los estudios sobre el consumo cultural, más que encuestas de opinión, son interpretaciones de datos con fines estratégicos que nos ofrecen una visión del consumidor cultural, acercándonos a su forma de 
pensar, a sus actitudes ante el consumo de productos y servicios culturales ofrecidos; bien sea por la administración pública que dirige las políticas culturales nacionales, o por el sector privado empresarial.

Potencialmente, la realización de encuestas de consumo cultural en el espacio geográfico cultural latinoamericano vienen a ser herramientas estratégicas de formulación y evaluación de políticas públicas culturales, indispensables dentro del delicado proceso implícito en la toma de decisiones para la inversión cultural, pues basado en el conocimiento que se tenga del consumidor, de la oferta y demanda, se estará en capacidad de dar un enfoque más preciso a los planes y programas de desarrollo cultural.

En este sentido, conocer el perfil del consumidor cultural latinoamericano es identificar sus cualidades culturales, ante aspectos particulares y específicos, a través de una serie de tópicos relacionados con sus actividades, intereses y opiniones, con el fin de configurar patrones de usos y apropiación simbólica, y lo más importante: advertir la "demanda cultural y la formación de públicos" en nuestro espacio geográfico cultural.

\section{Referencias}

Bernat, L. F., Mora, J. J., y Zuluaga, B. (2012). La elasticidad ingreso del consumo cultural en Cali. Revista de Economía Institucional, 27(14), 165-192.

Blondet Hernández, R. A. (2015). Informe de cobertura del análisis de resultados de la Encuesta Nacional de Consumo Cultural ENCC-RD-2014. República Dominicana. Coordinación Educativa y Cultural Centroamericana. Sistema de Integración Centroamericano (CECC-SICA). Mimeografiado.

Catalán, C., y Sunkel, G. (1992). Algunas tendencias en el consumo de bienes culturales en América Latina. Documento de Trabajo Flacso. Programa Chile. Serie: Educación y Cultura N. ${ }^{\circ}$ 27. Santiago de Chile.

Catalán, C., y Torche, P. (Eds.). (2005). Consumo cultural en Chile: miradas y perspectivas. Santiago de Chile: Consejo Nacional de la Cultura y las Artes, Instituto Nacional de Estadísticas y La Nación.

Cepal. (2014). Cultura y desarrollo económico en Iberoamérica. Madrid: Organización de Estados Iberoamericanos para la Educación, la Ciencia y la Cultura (OEI).

Consejo Nacional de la Cultura y las Artes de Chile. (2003). Impacto de la cultura en la economía chilena: participación de algunas actividades culturales en el PBI y evaluación de las fuentes estadísticas disponibles. Colección Economía y Cultura. Bogotá: Consejo Nacional de la Cultura y las Artes de Chile/Universidad ARCIS/ Convenio Andrés Bello (CAB). 
CAB - Convenio Andrés Bello. (2009). Cuentas satélites de cultura. Manual metodológico para su implementación en Latinoamérica. Bogotá: Convenio Andrés Bello. Agencia Española de Cooperación Internacional para el Desarrollo (AECID).

Ceicos-CorporaciónCentrosdeEstudios, Investigación y Comunicación Social. (1998). Consumo cultural en Bogotá. Experiencias vitales y estéticas: actuar, sentir y pensar. Bogotá: Ceicos y Observatorio de Cultura Urbana.

DANE - Departamento Administrativo Nacional de Estadísticas. (2007). Metodología de la Cuenta Satélite de Cultura. Colección Documentos N. ${ }^{\circ} 43$. Bogotá: DANE/Dirección de Síntesis y Cuentas Nacionales (DSCN).

Frey, B. (2000). La economía del arte. Colección Estudios Económicos N. 18. Barcelona: Editorial La Caixa/Caja de Ahorros y Pensiones de Barcelona.

García Canclini, N. (1990). Culturas híbridas. Estrategias para entrar y salir de la modernidad. Colección Los Noventa. México, D. F.: Coedición Dirección General de Publicaciones del Consejo Nacional para la Cultura y las Artes/ Editorial Grijalbo.

García Canclini, N. (Coord.). (1993). El consumo cultural en México. Colección Pensar la Cultura. México, D. F.: Dirección General de Publicaciones del Consejo Nacional para la Cultura y las Artes.

García Canclini, N. (1999). El consumo cultural: una propuesta teórica. En
G. Sunkel (Coord.), El consumo cultural en América Latina, pp. 26-49. Bogotá: Convenio Andrés Bello.

Gayo, M. (Dir.), Méndez, M. L., Radakovich, R., y Wortman, A. (2011). Consumo cultural y desigualdad de clase, género y edad: un estudio comparado en Argentina, Chile y Uruguay. Serie Avances de Investigación N.- 62. Madrid: CeALCI-Fundación Carolina.

Guzmán Cárdenas, C. E. (2005). La dinámica de la cultura en Venezuela y su contribución al PIB. Colección Economía y Cultura N. 10. Bogotá: Ministerio de Educación, Cultura y Deportes de Venezuela, Viceministerio de Cultura, CONAC, Convenio Andrés Bello.

Guzmán Cárdenas, C. E. (2009). Explorando las industrias creativas, de la experiencia y culturales. Anuario Ininco/Investigaciones de la Comunicación, 21(1), 117-173. Caracas: Universidad Central de Venezuela. Instituto de Investigaciones de la Comunicación. Facultad de Humanidades y Educación.

Guzmán Cárdenas, C. E. (2012). El valor de la cultura. Contribución de la Economía de la cultura. Anuario Inincol Investigaciones de la Comunicación, 24(1), 193-235. Caracas: Universidad Central de Venezuela. Instituto de Investigaciones de la Comunicación. Facultad de Humanidades y Educación.

Guzmán Cárdenas, C. E. (2013). Economía y política cultural en Venezuela. Revisión y perspectivas. Anuario ININCO/Investigaciones de la 
Comunicación, 25(1), 225-270. Caracas: Universidad Central de Venezuela. Instituto de Investigaciones de la Comunicación. Facultad de Humanidades y Educación.

Guzmán Cárdenas, C. E. (2014). Economía de la cultura y de la creatividad. Contratexto, 22, pp. 231-269.

Guzmán Cárdenas, C. E. (2015). El consumo cultural. La experiencia latinoamericana y República Dominicana. En Organización de Estados Iberoamericanos para la Educación, la Ciencia y la Cultura. Encuesta Nacional de Consumo Cultural de la República Dominicana (ENCC-RD 2014). Santo Domingo, República Dominicana: OEI.

Herrera Prieto, L. C. (2002). La economía de la cultura en España: una disciplina incipiente. Revista Asturiana de Economía, 23, 147-175.

Herrera Prieto, L. C. (2009). La investigación en economía de la cultura en España: un estudio bibliométrico. Revista Estudios de Economía Aplicada, 27(1), 35-62.

Herrera-Usagre, M. (2011). El consumo cultural en España. Una aproximación al análisis de la estratificación social de los consumos culturales y sus dificultades metodológicas. Empiria. Revista de Metodología de Ciencias Sociales, 22, 141-172.

Mantecón, A. R. (2002). Los estudios sobre consumo cultural en México. En D. Mato (Coord.), Estudios y otras prácticas intelectuales latinoamerica- nas en cultura y poder, pp. 255-263. Caracas: Clacso/CEAP/FACES UCV.

Martín-Barbero, J. (1987). De los medios a las mediaciones. Comunicación, cultura y hegemonía. (GG MassMedia). México, D. F.: Gustavo Gili.

Martín-Barbero, J. (1999). Recepción de medios y consumo cultural: travesías. En G. Sunkel (Coord.), El consumo cultural en América Latina, pp. 2-25. Bogotá: Convenio Andrés Bello.

Martín-Barbero, J., y Téllez, M.P. (2006). Los estudios de recepción y consumo en Colombia. Diálogos de la Comunicación, 73, 57-69. Lima: Federación Latinoamericana de Facultades de Comunicación Social-Felafacs.

Ministerio de Cultura de Colombia/ Convenio Andrés Bello. (2003). Impacto económico de las industrias culturales en Colombia. Colección Economía y Cultura. Bogotá: Ministerio de Cultura de Colombia/ Convenio Andrés Bello (CAB).

OEI-Organización de EstadosIberoamericanos para la Educación, la Ciencia y la Cultura. (2014). Encuesta latinoamericana de hábitos y prácticas culturales. Madrid: OEI.

OEI - Organización de Estados Iberoamericanos para la Educación, la Ciencia y la Cultura. (2015). Encuesta Nacional de Consumo Cultural de la República Dominicana (ENCC-RD 2014). Santo Domingo, República Dominicana: OEI.

Orozco, G. (1999). Televidencia y mediaciones. La construcción de estrategias por la audiencia. En G. 
Sunkel (Coord.), El consumo cultural en América Latina, pp. 68-86. Bogotá: Convenio Andrés Bello.

Retis Rivas, J. (2011). Estudio exploratorio sobre el consumo cultural de los inmigrantes latinoamericanos en España: el contexto transnacional de las prácticas culturales. Madrid: Fundación Alternativas/Observatorio Cultura y Comunicación.

Stolovich, L., Lescano, G., y Mourelle, J. (1997). La cultura da trabajo. Entre la creación y el negocio: economía y cultura en el Uruguay. Montevideo: Fin de Siglo.

Sunkel, G. (1999). (Coord.). El consumo cultural en América Latina. Bogotá: Convenio Andrés Bello.
Sunkel, G. (2002). Una mirada otra. La cultura desde el consumo. En D. Mato (Coord.), Estudios y otras prácticas intelectuales latinoamericanas en cultura y poder, pp. 287-294. Caracas: Clacso/CEAP/FACES/UCV.

Throsby, D. (2008). Economía y cultura. México, D. F.: Consejo Nacional para la Cultura y las Artes.

Villanueva Güell, P., Peters Núñez, T., y Morales Olivares, R. (2010). Una canasta básica de consumo cultural para América Latina. Elementos metodológicos para el derecho a la participación cultural. Santiago de Chile: Convenio Andrés Bello/Centro de Investigaciones Socioculturales (Cisoc) de la Universidad Alberto Hurtado de Santiago de Chile. 